\title{
Sistemas de significados en torno a la experiencia erótica y los abusos sexuales en la infancia*
}

\author{
Systems of Meanings concerning the Erotic Experience \\ and Child Sexual Abuse
}

\author{
Alma Vanessa Guzmán Díaz* \\ ORCID: 0000-0003-4839-972X \\ Patricia Trujano Ruíz \\ ORCID: 0000-0003-4956-8724
}

Universidad Nacional Autónoma de México

Recibido: 12 de noviembre de 2018

Revisado: 7 de julio de 2019

Aceptado: 30 de septiembre 2019

\section{Resumen}

El abuso sexual infantil puede dejar efectos en la experiencia erótica de las personas. Sin embargo, cuando dicha vivencia es narrada por los participantes ellos podrían significar de diversas maneras sus posibles consecuencias. Por lo tanto, llevamos a cabo una investigación cualitativa, bajo el marco teórico del construccionismo social, en la que participaron cuatro mujeres y dos hombres, quienes experimentaron abusos sexuales en la infancia. Realizamos entrevistas semi-estructuradas y temáticas. En el análisis de las narrativas, observamos la importancia de los constructos de género tradicionales en las primeras experiencias eróticas. Adicionalmente, las vivencias de abusos sexuales permitieron dar sentido a las dificultades observadas en la edad adulta. Finalmente, destacamos que el valor otorgado al amor fue un elemento que permitió la resignificación positiva de la experiencia.

Palabras clave: abuso sexual infantil; erotismo; construccionismo social; narrativa; investigación cualitativa.

Artículo de investigación. Citar como: Guzmán, D. A. \& Trujano, R. P. (2020). Sistemas de significados en torno a la experiencia erótica y los abusos sexuales en la infancia. Diversitas: Perspectivas en Psicología, 16(1), 65-77. Dol: https://doi.org/10.15332/22563067.3952

Para la realización de la investigación, la primera autora fue becada en México por el Consejo Nacional de Ciencia y Tecnología, con número de becario 211115, entre el periodo de 2014 al 2018.

Autor de correspondencia: Alma Vanessa Guzmán Díaz, Universidad Nacional Autónoma de México, Programa de Maestría y Doctorado en Psicología, Facultad de Estudios Superiores Iztacala. Dirección postal: Av De Los Barrios Número 1, Col. Los Reyes Iztacala, Tlalnepantla, Estado de México, C.P. 54090. Correo electrónico: alma.guzman.diaz@comunidad.unam.mx 


\section{Abstract}

Child sexual abuse may have effects on the erotic experience of people. However, when such an episode is narrated by the participants, they might give meaning to its possible consequences in different ways. Therefore, we carried out a qualitative research within the theoretical framework of social constructionism involving four women and two men who experienced sexual abuse in childhood. We conducted semi-structured and thematic interviews. In the analysis of the narratives, we noted the importance of the traditional gender constructs in the first erotic experience. Additionally, the experiences of sexual abuse helped to make sense of the difficulties observed in adulthood. Finally, we highlight that the value attributed to love was an element that allowed the positive re-signification of the experience.

Keywords: child sexual abuse; eroticism; social constructionism; narrative, qualitative inquiry.

\section{Introducción}

El presente trabajo entiende a los abusos sexuales en la infancia como experiencias fundamentalmente sociales dentro de una matriz de factores relacionales que las hacen posibles (Gibson \& Morgan, 2013; Magnabosco, 2014; Stainton, Stainton, \& Musitu, 1994). Entre estos factores podemos mencionar la representación de la infancia en sociedades occidentalizadas como un periodo de inocencia e ignorancia (Burman, 2003), particularmente sobre temas sexuales (Malón, 2011). En nuestra cultura, esta percepción puede colocar a niñas y niños en un estado de vulnerabilidad frente a los adultos que ejercen su poder -emanado de una mayor jerarquíasobre las y los menores, especialmente para utilizar sexualmente sus cuerpos a través de mentiras, engaños o amenazas (Krug, Dohlberg, Mercy, Zwi, \& Lozano, 2003; Orjuela \& Rodríguez, 2012).

Al respecto, se ha estimado que a nivel mundial el $20 \%$ de las mujeres y el $10 \%$ de los hombres habrían experimentado abusos sexuales durante su infancia (World Health Organization, 2014). Sin embargo, la vivencia de los hombres ha sido menos estudiada que la de las mujeres (Jackson, Newall, \& BackettMilburn, 2015). Este fenómeno puede mostrar diferentes efectos en las vidas de las personas, observando una diversidad de posibles consecuencias físicas, psicológicas, cognitivas, comportamentales, relacionales, funcionales, emocionales, y sexuales (Pereda, 2009, 2010).

En relación con los efectos de tipo sexual, se ha asociado la experiencia del abuso sexual en la infancia con comportamientos sexuales problemáticos ${ }^{1}$; por ejemplo conductas sexualmente intrusivas sobre los pares (Latzman \& Latzman, 2015). También se ha relacionado con un elevado interés frente a temas sexuales y con altos índices de ansiedad ante los mismos (Simon \& Feiring, 2008). En la adultez, se han reportado interacciones entre los antecedentes de abusos y las conductas sexuales de riesgo (González, Troncoso, Molina, \& Martínez, 2014), así como con mayores probabilidades de infecciones de transmisión sexual (González-Pacheco, Lartigue, \& Vázquez, 2008). Igualmente, se ha asociado con disfunciones sexuales femeninas en las fases de excitación y en la capacidad para alcanzar el orgasmo (Pérez, 2009), y se ha identificado como un indicador del trastorno del orgasmo masculino (Sánchez, Carreño, Corres, \& Henales, 2010), y del deseo sexual hipoactivo (Sánchez, Corres, Blum, \& Carreño, 2009).

Otros estudios cualitativos y con un enfoque narrativo han mostrado que cuando las personas relatan experiencias como los abusos sexuales, así como las formas en que los han enfrentado, se pueden encontrar diversos significados y mayor complejidad en las construcciones de sentido alrededor de la vivencia (Gibson \& Morgan, 2013). Desde esta perspectiva, la investigación se ha concentrado principalmente en la exploración de los significados en torno a los abusos y la identidad (Krayer, Seddon, Robinson, \&

$1 \quad$ El término se refiere a conductas iniciadas por niños menores de 12 años que involucran partes del cuerpo sexuales (por ejemplo los genitales, el ano, los senos), que culturalmente se consideran inapropiadas para la etapa de desarrollo y/o potencialmente dañinas para los niños involucrados (Latzman \& Latzman, 2015). 
Gwilym, 2015; Saha, Chung, \& Thorne, 2011); o en la identificación de las emociones asociadas con la experiencia (Foster \& Hagedorn, 2014). Pero se han abordado tangencialmente las interpretaciones que los participantes narran, específicamente sobre los abusos sexuales y la experiencia de atracción y de placer sexual.

Al respecto, en el presente estudio proponemos integrar estos dos elementos bajo el término de experiencia erótica. En primer lugar, entendemos a la experiencia como lo que hacemos en la participación relacional, más allá de comprenderla como una posesión privada de los sujetos (Gergen \& Gergen, 2011). En segundo lugar, aunque concebimos lo erótico como una manifestación personal, esta se encuentra enmarcada en esquemas culturales situados social e históricamente, que indican quién y qué partes del cuerpo son atractivas (Peña \& Hernández, 2015); así como dónde, cuándo, cómo y para qué se produce placer sexual en los individuos (Gagnon, 1980).

Adicionalmente, partimos del supuesto de que se pueden observar los sistemas de significado o el conjunto de creencias, valores y voces significativas -entendidas como puntos de vista de otras personas que "ayuden a contrarrestar el monólogo negativo, creando a su vez un diálogo abierto y más positivo" (Limón, 2012, p. 92)- en las narrativas que las personas construyen en torno a su experiencia erótica. En sociedades occidentalizadas es de esperarse que las formas de pensar, sentir y actuar eróticamente de los individuos están relacionadas con las cons- trucciones de género tradicionales que norman las formas de experimentar el erotismo femenino y masculino (Sanz, 1991).

Por lo tanto, llevamos a cabo una investigación de corte cualitativo desde el marco teórico del construccionismo social, a través del análisis de las narrativas producidas con las y los participantes (Gergen \& Gergen, 2011). El objetivo de este trabajo fue explorar y analizar los sistemas de significados alrededor de los efectos del abuso sexual en la infancia en su experiencia erótica en los discursos de mujeres y hombres.

\section{Método}

\section{Participantes}

Participaron cuatro mujeres y dos hombres adultos habitantes de la Ciudad de México y del área metropolitana, cuyas características sociodemográficas se pueden observar en la Tabla 1. Los criterios utilizados para incluirles en el estudio fueron: (1) tener más de 18 años cumplidos; (2) haber vivido algún tipo de abuso sexual antes de dicha edad y (3) haber sido o ser consultantes de servicios psicológicos. Este último criterio estuvo relacionado principalmente con un cuidado ético, pero también partió del interés por conocer cómo se han transformado las experiencias de las personas a través del contacto con discursos terapéuticos y de la relación con profesionales en estos campos de conocimiento (McNamee, 2013).

Tabla 1.

Características de las y los participantes

\begin{tabular}{|c|c|c|c|c|c|c|}
\hline $\begin{array}{c}\text { Sexo/orientación } \\
\text { sexual }\end{array}$ & Edad & $\begin{array}{c}\text { Estado } \\
\text { civil }\end{array}$ & $\begin{array}{c}\text { Nivel } \\
\text { educativo }\end{array}$ & Tipo de abuso & $\begin{array}{c}\text { Quién ejerció el } \\
\text { abuso }\end{array}$ & $\begin{array}{c}\text { Edad del } \\
\text { abuso }\end{array}$ \\
\hline Mujer, heterosexual & 39 & Soltera & Técnico & $\begin{array}{l}\text { Tocamientos, } \\
\text { exhibicionismo }\end{array}$ & $\begin{array}{l}\text { Padre adoptivo, } \\
\text { vecino }\end{array}$ & 7 a 14 \\
\hline Mujer, heterosexual & 37 & Soltera & Licenciatura & Penetración & Tío paterno & 5 a 8 \\
\hline Mujer, heterosexual & 47 & Casada & Licenciatura & Tocamientos & Tío político, vecinos & 4 a 7 \\
\hline Mujer, heterosexual & 41 & Casada & Licenciatura & Penetración & Padre biológico & 11 a 13 \\
\hline Hombre, homosexual & 31 & Soltero & Licenciatura & Penetración & Hermano & 5 y 12 \\
\hline Hombre, heterosexual & 25 & Soltero & Bachillerato & Penetración & Amigo de la familia & 13 a 17 \\
\hline
\end{tabular}

Fuente: elaboración propia. 
En la Tabla 1 también se muestran las formas de abusos sexuales que las y los participantes vivieron durante la infancia o adolescencia. En todos ellos las situaciones de abuso ocurrieron en más de una ocasión, de forma permanente o intermitente. En algunos casos fueron ejercidos por diferentes personas, dentro y fuera de la familia-, en distintos momentos antes de cumplir los dieciocho años, en espacios tanto privados como públicos. Además, cada persona señaló las edades aproximadas de inicio y término de estos eventos.

Para tener acceso a las y los participantes contamos con el apoyo de un programa universitario de atención a la violencia, así como con la colaboración de terapeutas que les estaban atendiendo -en consulta privada- o lo habían hecho en el pasado. En este punto, no sólo nos interesamos en explorar los discursos terapéuticos, sino que era importante para nosotras saber que las personas entrevistadas habían narrado estas vivencias al menos en una ocasión y que lo habían hecho en un entorno de acompañamiento y con profesionales. Por último, el protocolo del estudio fue revisado y aprobado antes de comenzar el trabajo de campo -las entrevistaspor un comité conformado por cuatro investigadores, tres formaban parte de nuestra universidad y uno externo.

\section{Procedimiento}

Se llevaron a cabo entrevistas semi-estructuradas (Rivas, 2010), enfocadas en los siguientes ejes temáticos: la experiencia erótica en la infancia, las situaciones de abuso sexual, los procesos psicoterapéuticos, la experiencia erótica en la actualidad y hacia el futuro. Además, se leyó, aprobó y firmó -con cada participante- el documento del consen- timiento informado que incluyó los objetivos del estudio, las condiciones para llevarlo a cabo y la confidencialidad de sus identidades. La sesión tuvo un promedio de dos horas y fue realizada individualmente en un aula libre de distractores. Los discursos fueron audiograbados y posteriormente transcritos.

En el análisis de las narrativas -el cual llevó a cabo la primera autora- realizamos una lectura inicial de la transcripción de cada una de las entrevistas, marcamos las partes que correspondían a los cuatro ejes temáticos antes mencionados y nos enfocamos en identificarlas con códigos (Riessman, 2008) destacando cuatro categorías, cada una con distinto número de niveles y subniveles. Estos indicaron los elementos que compartieron o difirieron entre los relatos de las y los participantes.

Posteriormente, para ejemplificar cada una de las categorías elegimos casos particulares -de aquí en adelante segmentos- para ilustrar los patrones o variaciones de las observaciones; igualmente, presentamos nuestras interpretaciones e hipótesis sobre estas, de acuerdo con la literatura revisada. Estos segmentos preservaron la secuencia de los relatos de los cuales fueron extraídos.

\section{Resultados}

En el análisis de las narrativas observamos cuatro categorías, entre estas elegimos dos -que mostramos en la Tabla 2- porque permiten observar claramente la complejidad de la experiencia erótica, así como la diversidad de interpretaciones y la transformación de significados (Martínez-Guzmán \& Montenegro, 2014), con respecto a los posibles efectos de los abusos sexuales. 
Tabla 2.

Categorías en torno a los efectos del abuso sexual en la experiencia erótica.

\begin{tabular}{|c|c|c|}
\hline Categoría & Niveles & Subnivel \\
\hline \multirow{2}{*}{$\begin{array}{l}\text { Experiencia erótica } \\
\text { durante la niñez }\end{array}$} & $\begin{array}{l}\text { Primeras } \\
\text { exploraciones }\end{array}$ & $\begin{array}{l}\text { Hombres: } \\
\text { Experimentación } \\
\text { Límites heterosexuales } \\
\text { Mujeres: } \\
\text { Ausencia de experiencias }\end{array}$ \\
\hline & Primeras atracciones & $\begin{array}{l}\text { Hombres: } \\
\text { Permisividad } \\
\text { Mujeres: } \\
\text { Atracción y vigilancia } \\
\text { Mujeres y hombres } \\
\text { Inhibición por abuso }\end{array}$ \\
\hline \multirow[t]{2}{*}{$\begin{array}{l}\text { Interpretación de los } \\
\text { efectos de los abusos } \\
\text { sobre la experiencia } \\
\text { erótica }\end{array}$} & $\begin{array}{l}\text { En la atracción } \\
\text { y el placer }\end{array}$ & $\begin{array}{l}\text { Hombres: } \\
\text { Miedo } \\
\text { Sobre-estimulación } \\
\text { Mujeres: } \\
\text { Desagrado } \\
\text { Recuerdos del abuso } \\
\text { Experimentar con varias parejas } \\
\text { Intercambio por afecto } \\
\text { Mujeres y hombres: } \\
\text { Violencia sexual }\end{array}$ \\
\hline & Re-significación & Construcción del amor \\
\hline
\end{tabular}

Fuente: elaboración propia.

La Tabla 2 muestra cada una de las categorías, así como los niveles en los que se sub-clasificaron por sexo, con excepción del último subnivel. En el siguiente apartado se muestran segmentos de las entrevistas que ejemplifican cada una de las categorías.

\section{Experiencia erótica durante la niñez}

En esta categoría observamos dos subniveles. El primero fue denominado "las primeras exploraciones" y mostró diferencias por sexo. En el caso de los hombres, estas experiencias suelen llevarse a cabo entre pares a través de la enseñanza mutua de la exploración de sus cuerpos (Sapién \& Córdoba, 2011), las cuales fueron ubicadas por los participantes entre los 6 y 8 años. Para uno de ellos, las interacciones en las que él y otro niño se estimulaban entre sí ocurrieron antes del inicio de los abusos sexuales, estos contactos fueron narrados desde la curiosidad y la experimentación de sensaciones agradables. En palabras del entrevistado:

[...] alguna vez él me dijo 'mira esto que encontré' y le pusimos un poquito a la película [pornográfica] y pues empezamos a ver cómo tienen relaciones sexuales los mayores y nosotros dentro de esto mismo que nos acariciábamos pues también nos quitábamos la ropa y digamos que nos rozábamos este yo mi pene a sus nalgas y al revés ¿no? y yo sentía mucha excitación y él, pues él también (hombre, 25).

Sin embargo, observamos el mandato de la heterosexualidad y el rechazo al deseo homoerótico (Cruz, 2014) en la narrativa del otro participante. El entrevistado, quien había vivido abusos sexuales a los cinco años, narró que en la etapa de la educación primaria intentó dar un abrazo a un niño que le atraía, haciéndolo en el salón de clases frente a otros amigos; sin embargo, fue sancionado por el 
otro sujeto, quien le señaló que este tipo de interacción entre varones le resultaba desagradable, como lo narró el entrevistado:

[...] entonces lo que sí recuerdo fue que respiré y como que lo abracé, pero fue esa manera en la que lo abracé y yo... pues lo olí, que su respuesta fue agarrarme y dijo 'iah! este, no vuelvas a hacer eso' y yo dije ¿por qué? [...] ¿por qué no te gusta?, yo ahora supongo que yo habré dicho 'si esto fue tan bonito o tan rico para mí, ¿por qué no te gustó?' y recuerdo que él me dijo 'no sé cómo explicarte, no sé cómo decírtelo, pero no me gusta' entonces le dije ‘jah! bueno, está bien' y ya seguimos siendo amigos (hombre, 31 ).

En contraste, en el caso de las mujeres observamos la ausencia de relatos sobre exploraciones del propio cuerpo o con pares. Al respecto de este vacío en la experiencia erótica femenina en la niñez, Lagarde (2005) ha argumentado que el cuerpo de las niñas suele ser tocado sólo con fines de limpieza o embellecimiento, pero sin intencionalidad erótica. También se ha señalado que cuando se entrevista a mujeres adultas, estas podrían reservarse el compartir estas vivencias eróticas por sentir vergüenza o porque consideran que son cuestiones que les corresponden únicamente a los hombres (Estrada, Wong, Pérez, \& Pacheco, 2008). Aunado a lo anterior, cabe subrayar que en todos los casos, las entrevistadas situaron el inicio de los abusos sexuales entre los 4 y 11 años, el periodo culturalmente asignado a la infancia, lo cual pudo haber inhibido aún más la posibilidad de exploración de sus propios cuerpos o de otros.

Con respecto a las "primeras atracciones", en la narrativa de los varones advertimos la permisividad social en la búsqueda del éxito con las mujeres, así como mayor número de conquistas y libertad de acción para llevarlas a cabo (Salguero, 2014). Uno de los participantes relató su vivencia, la cual fue ubicada temporalmente antes del inicio de los abusos sexuales. En sus palabras:

"en primero de secundaria es cuando tengo mi primera novia, de decirle '¿oye quieres ser mi novia?' y 'sí vamos a ser novios' y nos besamos y todo [...] también era como parte de un juego porque yo así les decía a muchas chicas no sólo a ella, les decía ¿oye quieres ser mi novia? pero no lo decía en serio" (hombre, 25).

La experiencia de tres mujeres entrevistadas estuvo enmarcada en las creencias tradicionales que aún valoran la virginidad de las jóvenes (Amuchástegui, 1998). Al parecer, para las participantes la atracción se vivió en un contexto en el cual algunos adultos, especialmente los hermanos y el padre, estaban encargados de vigilarlas. Una de estas mujeres relató la forma en que experimentó su primer beso:

"no me dejaban sola ni tantito para que yo tuviera contacto con el niño este, total que me empieza a gustar el niño, y una vez salí y él me alcanzó y me dijo 'oye me gustas ¿quieres ser mi novia?’ y yo ¡no! ¡no!, me agarró y me dio un beso, pero en ese momento llegó mi hermano, no sabes cómo me fue, me regañaron, me pegaron, me gritonearon y me castigaron, si de por sí no salía, pues nada más lo veía por la ventana, y decía pero ¿por qué? no tiene nada de malo" (mujer, 39).

Hasta este punto, en las narrativas de dos de las mujeres y de uno de los varones no observamos asociaciones directas o problemáticas entre las primeras exploraciones, las atracciones durante la infancia y los abusos sexuales, los cuales ya habían experimentado en la niñez, cuya relación se ha intentado establecer con frecuencia en la literatura (Latzman \& Latzman, 2015; Simon \& Feiring, 2008). No obstante, otras dos mujeres y uno de los varones narraron experiencias de "inhibición de la atracción" que ubicaron aparentemente en la misma época en que estaban ocurriendo los abusos sexuales. En las palabras de una de las mujeres:

"ese abuso [por parte de su padre] yo siento que también me castró y me cambió la vida, porque yo cuando veía ese niño yo sentía tan bonito en mi cuerpo, yo sentía mariposas en el estómago, que los pelitos de la piel se me paraban de punta, que el corazón me latía más rápido y ese niño creo que ni sabía que existía, pero yo nada más con verlo yo en ese pueblo era feliz, era así como un algo que me hacía sentir tan bonito, pero ya cuando pasó eso [el abuso sexual]... ya nunca jamás lo busqué" (mujer, 41). 
Mientras que el hombre entrevistado dijo:

"la palabra es que fue removido, creo que el resultado fue eso, la inhibición de alguna u otra manera, aunque sí había niños, por ejemplo, niños que me gustaban" (hombre, 31).

\section{Interpretaciones de los efectos de los abusos sobre la experiencia erótica}

En la literatura se ha planteado que las personas que han pasado por este tipo de experiencia podrían establecer asociaciones entre recuerdos temerosos del evento del abuso con la actividad sexual; así como otorgar significados erróneos o distorsionados a partes del cuerpo o a la conducta sexual (Finkelhor \& Browne, 1985). En esta categoría identificamos la presencia de los elementos mencionados así como de otros adicionales, y advertimos que los sentidos otorgados a estos difirieron en las historias construidas por las mujeres y por los hombres.

En uno de los relatos de los varones observamos un conflicto entre la atracción homoerótica que él experimentaba y la posibilidad de llevarla a cabo, que más allá de las creencias heterosexistas (Rubin, 1989), fue explicado a través del miedo que el participante sentía hacia las personas del sexo de quien lo agredió en la infancia, como él mismo lo contó:

"fue realmente cuando troné, porque fue como el conflicto de isí, lo sé! me gustan los hombres, esta es mi identidad sexual, pero fue iputa madre es un hombre! ¿qué voy a hacer con eso? ¡me da miedo! ¿cómo me voy a relacionar con él? y creo que ese conflicto fue tan grande que haz de cuenta que a partir de ahí los hombres me asustaban, todos los hombres me asustaban" (hombre, 31).

Mientras que un segundo participante identificó como una dificultad la sobreestimulación del pene, que de acuerdo con Sanz (1991) ha sido una parte del cuerpo de los hombres en la cual suele centrarse el erotismo masculino. En el caso analizado, las características del abuso sexual -llevado a cabo por otro hombre- al parecer permitieron al entrevistado construir una explicación coherente con respecto a las sensaciones corporales enfocadas en la parte que fue estimulada en el pasado, en sus palabras:

"tuvo muchísimos efectos, por ejemplo como el abuso era muy constante, no solamente constante en cuanto a días, sino a veces dentro del mismo día abusaba dos tres veces de mí, yo pues quedo pues como sobre estimulado [...] y pues así como haciendo como una separación de lo que era antes a lo que era después, puedo ver que eso es algo que pasa después [...] se me hace una necesidad muy muy grande el sentir la estimulación sexual en mi cuerpo, sobre todo del pene" (hombre, 25).

En la experiencia de dos mujeres entrevistadas más allá de asociaciones con recuerdos temerosos (Finkelhor \& Browne, 1985), la vivencia se significó a través del desagrado en la experiencia erótica, la cual además tomó sentido una vez que el significado del abuso sexual estuvo disponible como una de las posibilidades para narrar las sensaciones corporales displicentes, como explicó una de las participantes:

"el abuso sexual fue entre los cuatro y los siete años, más o menos y eso estuvo bloqueado hasta los veintinueve años [...] entonces en este lapso de adolescencia que yo estaba renuente a una relación física, yo no sabía por qué, yo tenía esa molestia de que se acercaran o de que me tocaran o de tener relaciones" (mujer, 47).

Adicionalmente, en la literatura se han tratado de establecer asociaciones entre el número de parejas y el antecedente de abuso sexual, aunque sin poder corroborarlo (Simon \& Feiring, 2008). En nuestro estudio, dos participantes expresaron haber pasado por periodos o etapas de "experimentación sexual con varias parejas". Por ejemplo, una de ellas tuvo sexo con hombres distintos el mismo día en una fase de su vida, mientras que otra participante narró que con algunas parejas se involucró afectivamente y con otras no lo hizo, en sus palabras:

"llegó un momento, en que quiero pensar que es porque traigo este trauma [por el abuso sexual] o no sé cómo llamarlo, empecé a buscar como relaciones en páginas como de Facebook y así, como de hacer amigos y así, yo empecé a salir con muchos... con algunos sí tuve relacio- 
nes, con otros no, pero era de bueno pues tú me gustas pues ¡sí! tú no ¡bye!” (mujer, 39).

Con respecto a la conducta sexual como una estrategia para intercambiar bienes, para manipular a otros o para satisfacer las necesidades propias que los menores podrían aprender como resultado del abuso (Finkelhor \& Browne, 1985), observamos que dos participantes narraron que en la adultez ellas podrían haber intercambiado sexo principalmente para llenar necesidades de carácter afectivo, como lo ejemplificamos con las palabras de una mujer:

"yo siento que era destrucción, yo siento que antes era pura destrucción, era a lo mejor tener relaciones para que no me dejaran, para que no me abandonaran, para pedir cosas, a lo mejor era para que me dieran atención, para que me dieran más atención, cariño" (mujer, 41).

Por otra parte, la literatura tradicional ha reportado también la revictimización sexual posterior como uno de los efectos a largo plazo del abuso sexual durante la infancia (Pereda, 2010). En efecto, en las narrativas del presente estudio advertimos que, en la adolescencia y la adultez, al menos dos mujeres y un hombre fueron forzados a tener relaciones sexuales. Una mujer dijo:

"me llevó a su casa [un novio], total que en su casa quiso abusar de mí, dije 'no, no quiero', es que sí que, ¡no! y ¡no! y ¡me lastimó!, y siempre me hizo creer que yo no lo quería, que me daba asco, que por eso no había querido tener relaciones con él" (mujer, 39).

Ahora bien, cuando las personas han experimentado situaciones recurrentes de violencia, pueden ver amenazado el sentido de sí mismas o de sus formas preferidas de ser y estar en el mundo (White, 2004). Por lo tanto, en el estudio exploramos si hubo elementos en el contexto de las personas entrevistadas que hubiesen ayudado a recuperar este sentido. En los relatos de dos mujeres y un hombre hubo referencias al valor del amor, que estuvieron relacionadas con la narración de relaciones con parejas en la edad adulta que se caracterizaron por brindar apoyo, construir confianza y mostrar paciencia para facilitar que las interacciones sexuales fuesen más satisfactorias, hasta llegar a experimen- tarse como placenteras. En palabras de uno de los participantes:

"para mí fue tan básico eso, los besos, los abrazos, pero yo creo fue ese amor que me dio, pero en el sentido de confianza [...] yo diría que hasta que interioricé que él realmente me amaba y que no me iba a hacer daño, fue cuando me pude abrir a tener relaciones sexuales con él, fue cuando realmente me sentí amado, cuando realmente me sentí en un contexto seguro" (hombre, 31).

Otra mujer lo explicó de la siguiente manera:

"fue una persona muy especial y no fue mi primera relación sexual jeh!... sí fue una persona muy especial una persona muy dulce... que pues primero me dio mucha seguridad y mucho cariño... antes de tener relaciones ¿no?, de hecho alguna vez íbamos a su casa y trabajábamos, leíamos, hacíamos cosas y nos divertíamos, y mucho tiempo no pasó nada [...] al final bueno nos empezamos a besar y las cosas fluyeron ¡súper bien! (mujer, 47).

De tal modo que en el discurso de las personas entrevistadas, el valor otorgado al amor destacó que la postura respetuosa y afectiva por parte de algunas de sus parejas sexuales, les permitió afrontar las dificultades en torno a la experimentación de placer sexual, que ellos mismos habían asociado -en sus narrativas- con las vivencias de abusos sexuales en edades tempranas.

\section{Discusión y Conclusiones}

El presente estudio tuvo como objetivo explorar y analizar los sistemas de significados alrededor de los efectos del abuso sexual en la infancia en la experiencia erótica en las narrativas de cuatro mujeres y dos hombres adultos, habitantes de la Ciudad de México y área metropolitana, quienes vivieron diferentes tipos de abusos sexuales durante la infancia, como se mostró en la Tabla 1, por parte de familiares y de otras personas cercanas.

En las narrativas de las primeras experiencias eróticas de exploración y atracción en la infancia ob- 
servamos que especialmente los hombres relataron interacciones entre pares, las cuales son culturalmente aceptables en este periodo de vida (Sapién \& Córdoba, 2011). Por ejemplo, los participantes narraron vivencias de exploraciones de sus cuerpos llevadas a cabo con otros niños en espacios privados. Sin embargo, cuando contaron este tipo de acercamientos -entre hombres- en lugares públicos, las respuestas de los otros varones fueron de desagrado. Al parecer, los límites de la expresión del deseo homoerótico estarían regulados por los propios hombres socializados en el rechazo hacia cuerpos semejantes (Cruz, 2014), lo cual puede reflejar el heterosexismo de nuestra cultura.

Como se esperaba, los varones -en comparación con las mujeres- mostraron que disponían de mayor número de oportunidades para experimentar el acercamiento con otras personas como parte de la construcción de su masculinidad (Salguero, 2014). Mientras que para las mujeres no observamos este tipo de experiencias en ninguno de los espacios mencionados. Estas diferencias coinciden con lo descrito por Lagarde (2005) con respecto a que nuestra cultura suele promover una mayor experimentación del erotismo masculino, así como que este todavía se aprende entre varones a través de prácticas homoeróticas (Sapién \& Córdoba, 2011).

Las experiencias de atracción en los espacios públicos en los relatos de las mujeres estuvieron enmarcadas por la vigilancia, el castigo y la moderación de los adultos. Al parecer, existen creencias que sostienen que el espacio público es más peligroso para las mujeres y se les debe cuidar, mientras que los espacios privados -como el hogar- son significados como sitios seguros para ellas (Soto, 2013), aunque es en estos últimos en donde suelen ocurrir los abusos sexuales por personas conocidas (Frías \& Erviti, 2014; Pineda-Lucatero, Trujillo-Hernández, Millán-Guerrero, \& Vásquez, 2008)Los relatos de los participantes varones mostraron que la experimentación erótica podría iniciar desde edades entre los 6 y 8 años, que son más tempranas que el rango de los 11 y 13 años que se ha reportado (Sapién \& Córdoba, 2011). Por otra parte, las mujeres narraron sus primeras experiencias de atracción y tocamientos -como el primer beso- alrededor de la edad promedio de los 14 años encontrada para poblaciones mexicanas (García-Rodríguez, 2013), lo cual puede reflejar su adhesión a guiones sexuales dominantes sobre el erotismo femenino.

En las narrativas sobre la experiencia erótica durante la infancia, las observaciones no incluyeron sobresexualización de las relaciones, ni conductas sexualmente intrusivas, tampoco un elevado interés por temas sexuales o altos niveles de ansiedad frente estos (Latzman \& Latzman, 2015; Simon \& Feiring, 2008). Contrariamente, un hombre y una mujer compartieron sensaciones de inhibición con respecto a la experimentación de atracción durante la infancia.

En la edad adulta, más allá de las descripciones de conductas sexuales de riesgo o disfunciones sexuales asociadas con el antecedente de abuso sexual infantil (González et al., 2014; González-Pacheco et al., 2008; Sánchez et al., 2009; Sánchez et al., 2010), las experiencias fueron caracterizadas por las personas (Vayreda, Tirado \& Domènech, 2005) como dificultades situadas en etapas o periodos de sus vidas, por ejemplo, el miedo al interactuar con personas del sexo de quien les agredió o el desagrado y evitación del contacto corporal o del intercambio de sexo por afecto.

Además, los significados que las personas otorgaron a los efectos de los abusos sobre su experiencia erótica pueden interpretarse como esfuerzos de los sujetos por recuperar un sentido de sí mismo -o las formas preferidas de ser y de sentir- que en ocasiones puede verse cuestionado en los sujetos que han pasado por la experiencia recurrente de violencia (White, 2004); así como para dar dirección y coherencia a los relatos sobre su identidad (Goolishian \& Anderson, 1998).

En las narrativas también observamos el valor del amor como un elemento del contexto que permitió resignificar la experiencia erótica de un sentido problemático hacia otro donde fuese más placentero. Por una parte, este hallazgo coincide con la literatura sobre erotismo femenino que ha reportado la asociación entre el amor como un factor que desencadena o motiva el deseo sexual en mujeres mexicanas (Cruz del Castillo, Romero, \& Gil-Bernal, 2013), lo anterior en una muestra que estuvo integrada por población general, sin considerar el antecedente de abusos sexuales en la infancia. 
De manera que, desde la investigación cualitativa y el enfoque narrativo, podemos señalar que las creencias culturales del género tradicionales aún guían las experiencias eróticas (Martínez-Guzmán \& Montenegro, 2014). Sin embargo, estas creencias no determinan la vida de las personas, puesto que son capaces de ejercer su potencial de agencia y transformar sus realidades (Gergen \& Gergen, 2011) al mostrar -en algunos de los relatos- que lograron ir más allá del discurso de la victimización, así como del daño permanente e irreversible a través del cual suelen describirse las vivencias del abuso sexual y de sus posibles efectos (Woodiwiss, 2014).

No obstante, es importante recordar que todas las personas entrevistadas tenían experiencia en contextos psicoterapéuticos, los cuales podrían haber influenciado la resignificación de las vivencias de abuso sexual, puesto que algunos de estos espacios suelen promover la reflexión constructiva (McNamee, 2013). En el estudio no contamos con participantes sin contacto con espacios de psicoterapia, por lo tanto, no podemos observar el alcance de otros posibles discursos o escenarios que también podrían ser relevantes.

Asimismo, señalamos la relevancia de considerar las diversas orientaciones sexuales de las personas y las posibles dificultades en la experiencia erótica en diferentes etapas de vida, puesto que en esta investigación observamos problemáticas entre la expresión del deseo sexual de las personas y que este se dirigiera hacia el mismo sexo del individuo que les agredió, como en el caso de un participante varón -con orientación homosexual- quien narró el conflicto en la atracción experimentada hacia individuos de su mismo sexo y que este coincidiera con el sexo de quien abusó sexualmente de él, lo cual le llevó a evitar interactuar con ellos.

Por lo tanto, sugerimos que para futuras investigaciones sean integradas poblaciones con características diferentes que permitan enriquecer la diversidad de narrativas analizadas. Entre las que proponemos incluir a personas sin experiencia en contextos psicoterapéuticos, a mujeres y varones con vivencias de abuso por parte de mujeres, a personas con diversas orientaciones sexuales, y a las redes de apoyo, la familia, círculos de amigos y los terapeutas, entre otras personas significativas.

\section{Referencias}

Amuchástegui, A. (1998). Saber o no saber sobre sexo: los dilemas de la actividad sexual femenina para jóvenes mexicanos. En I. Szasz \& S. Lerner (Comps.), Sexualidades en México: algunas aproximaciones desde la perspectiva de las ciencias sociales (100-127). México: El Colegio de México, Centro de Estudios Demográficos y de Desarrollo Urbano, Programa de Salud Reproductiva y Sociedad.

Burman, E. (2003). Chilhood, sexual abuse and contemporary political subjectivities. En P. Reavey \& S. Warner (Comps.), New feminist stories of child sexual abuse. Sexual scripts and dangerous dialogues (34-51). New York: Routledge.

Cruz, S. (2014). Prácticas corporales, erotismo y políticas de identidad: el caso del trabajo sexual masculino en la Ciudad de México. En T. Rocha, \& I. Lozano (Comps.), Debates y Reflexiones en torno a las masculinidades: Analizando los caminos hacia la igualdad de género (87-105). México: Facultad de Psicología, Universidad Nacional Autónoma de México.

Cruz del Castillo, C., Romero, A., \& Gil-Bernal, F. (2013). Indicadores del Deseo, Autoerotismo e Impulsividad Sexual en Mujeres de la Ciudad de México. Acta de Investigación Psicológica, 3(1), 1031-1040.

Estrada, S., Wong, S., Pérez, G., \& Pacheco, L. (2008). Las vivencias de autoerotismo de mujeres universitarias. Archivos Hispanoamericanos de Sexología, 14(2), 19-55.

Finkelhor, D. \& Browne, A. (1985). The traumatic impact of child sexual abuse: A conceptualization. American Journal of Orthopychiatry, 55(4), 530-541.

Foster, J. \& Hagedorn, B. (2014). A qualitative exploration of fear and safety with child victims of sexual abuse. Journal of Mental Health Counseling, 36(3), 243-262.

Frías, S. M. \& Erviti, J. (2014). Gendered experiences of sexual abuse of teenagers and children in 
Mexico. Child Abuse and Neglect, 38(4), 776-787. http://doi.org/10.1016/j.chiabu.2013.12.001

Gagnon, J. (1980). Sexualidad y cultura. México: Pax.

García-Rodríguez, G. (2013). Sexualidad femenina: expresiones del comportamiento erótico. En T. Rocha, \& C. Cruz del Castillo, Mujeres en transición: reflexiones teórico-empíricas en torno a la sexualidad, la pareja y el género (57-83). México: Universidad Iberoaméricana.

Gergen, K. \& Gergen, M. (2011). Narrative tensions. Perilous and productive. Narrative Inquiry, 21(2), 374-381.

Gibson, K. \& Morgan, M. (2013). Narrative Research on Child Sexual Abuse: Addressing Perennial Problems in Quantitative Research. Qualitative Research in Psychology, 10(3), 298-317. https:// doi.org/10.1080/14780887.2011.606597

González, E., Troncoso, P., Molina, T., \& Martínez, V. (2014). Antecedente de agresión sexual y su asociación con conductas de riesgo en adolescentes consultantes en un centro de atención para la salud sexual y reproductiva. Revista Chilena de Obstetricia Ginecológica, 79(1), 31-39.

González-Pacheco, I., Lartigue, T., \& Vázquez, G. (2008). Estudio de casos y controles con un grupo de mujeres embarazadas con experiencias adversas en la infancia y/o adolescencia e infecciones de transmisión sexual. Salud Mental, 31(4), 261-270.

Goolishian, H. \& Anderson, H. (1998). Narrativa y self. Algunos dilemas posmodernos de la psicoterapia. En D. Schnitman, Nuevos Paradigmas, Cultura y Subjetividad (293-306). Buenos Aires: Amorrortu.

Jackson, S., Newall, E., \& Backett-Milburn, K. (2015). Children's narratives of sexual abuse. Child \& Family Social Work, 20(3), 322-332. http://doi.org/10.1111/cfs.12080

Krayer, A., Seddon, D., Robinson, C. A., \& Gwilym, $\mathrm{H}$. (2015). The influence of child sexual abuse on the self from adult narrative perspectives.
Journal of Child Sexual Abuse, 24(2), 135-151. http://doi.org/10.1080/10538712.2015.1001473

Krug, E., Dohlberg, L., Mercy, J., Zwi, A., \& Lozano, R. (2003). Informe mundial sobre violencia y salud. Washington, D. C.: Organización Panamericana de la Salud.

Lagarde, M. (2005). Los cautiverios de las mujeres: madresposas, monjas, putas, presas y locas (4a. ed.). México: Universidad Nacional Autónoma de México.

Latzman, N. \& Latzman, R. (2015). Exploring the link between child sexual abuse and sexually intrusive behaviors: The moderating role of caregiver discipline strategy. Journal of Child Family Studies, 24(2), 480-490.

Limón, G. (2012). La terapia como diálogo hermenéutico y construccionista. Prácticas de libertad y deco-construcción en los juegos relacionales, de lenguaje y de significado [Online] Ohio: Taos Institute Publications. http://www.taosinstitute.net/Websites/taos/ images/PublicationsWorldShare/Gilberto_s_ Book__final.pdf

Magnabosco, M. (2014). El Construccionismo Social como abordaje teórico para la comprensión del abuso sexual. Revista de Psicología, 32(2), 220-242.

Malón, A. (2011). The "participating victim" in the study of erotic experiences between children and adults: An historical analysis. Archives of Sexual Behavior, 40(1), 169-188. http://doi. org/10.1007/s10508-009-9553-z

Martínez-Guzmán, A. \& Montenegro, M. (2014). La producción de narrativas como herramienta de investigación y acción sobre el dispositivo sexo/género: construyendo nuevos relatos. Quaderns de Psicología, 16(1), 111-125.

McNamee, S. (2013). Reconstrucción de la identidad; la construcción comunal de la crisis. En S. McNamee \& K. Gergen, La terapia como construcción (219-232). Barcelona: Paidós. 
Orjuela, L. \& Rodríguez, V. (2012). Violencia sexual contra los niños y las niñas. Abuso y explotación sexual infantil. Guía de material básico para la formación de profesionales. España: Save the Children.

Peña, E. \& Hernández, L. (2015). Entre cuerpos y placeres. Representaciones y prácticas sexuales en personas con discapacidad adquirida. Monterrey: Universidad Autónoma de Nuevo León.

Pereda, N. (2009). Consecuencias psicológicas iniciales del abuso sexual infantil. Papeles del Psicólogo, 30(2), 135-144.

Pereda, N. (2010). Consecuencias psicológicas a largo plazo del abuso sexual infantil. Papeles del Psicólogo, 31(2), 191-201.

Pérez, L. (2009). Funcionalidad sexual en mujeres sobrevivientes de abuso sexual en la infancia. México: Universidad Nacional Autónoma de México. Facultad de Medicina.

Pineda-Lucatero, A. G., Trujillo-Hernández, B., Millán-Guerrero, R. O. \& Vásquez, C. (2008). Prevalence of childhood sexual abuse among Mexican adolescents. Child: Care, Health and Development, 35(2), 184-189. http://doi. org/10.1111/j.1365-2214.2008.00888.x

Riessman, C. (2008). Narrative Methods for the Human Sciencies. California: Sage Publications.

Rivas, M. (2010). La entrevista a profundidad: un abordaje en el campo de la sexualidad. En I. Szasz \& S. Lerner (Comp.), Para comprender la subjetividad: investigación en salud reproductiva y sexualidad (199-223). México: El Colegio de México, Centro de Estudios Demográficos y de Desarrollo Urbano.

Rubin, G. (1989). Reflexionando sobre el sexo: notas para una teoría radical de la sexualidad. En C. Vence (Ed.), Placer y peligro. Explorando la sexualidad femenina (113-190). Madrid: Revolución.
Saha, S., Chung, C., \& Thorne, L. (2011). A narrative exploration of the sense of self of women recovering from childhood sexual abuse. Counselling Psychology Quarterly, 24(2), 101-113. http:// doi.org/10.1080/09515070.2011.586414

Salguero, M. A. (2014). Identidad masculina. Elementos de análisis en el proceso de construcción. México: Facultad de Estudios Superiores Iztacala, Universidad Nacional Autónoma de México.

Sánchez, C., Carreño, J., Corres, N., \& Henales, C. (2010). Perfiles e indicadores psicológicos relacionados con la dispaurenia y el vaginismo. Estudio cuantitativo Primera Parte. Salud Mental, 33(4), 347-353.

Sánchez, C., Corres, N., Blum, B., \& Carreño, J. (2009). Perfil de la relación de factores psicológicos del deseo sexual hipoactivo femenino y masculino. Salud Mental, 32(1), 43-51.

Sanz, F. (1991). Psicoerotismo femenino y masculino: Para unas relaciones placenteras y autónomas. Barcelona: Kairos.

Sapién, J. \& Córdoba, D. (2011). Diferencias sexuales entre hombres y mujeres. México: Facultad de Estudios Superiores Iztacala, Universidad Nacional Autónoma de México.

Simon, V. \& Feiring, C. (2008). Sexual anxiety and eroticism predict the development of sexual problems in youth with history of sexual abuse. Child Maltreatment, 13(2), 167-181.

Soto, P. (2013). Entre los espacios del miedo y los espacios de la violencia: discursos y prácticas sobre la corporalidad y las emociones. En M. A. Aguilar \& P. Soto (Coords.), Cuerpos, espacios y emociones. Aproximaciones desde las ciencias sociales (197-219). México: Universidad Autónoma Metropolitana, Unidad Iztapalapa; Miguel Ángel Porrúa.

Stainton, W., Stainton, R., \& Musitu, G. (1994). Abuso sexual infantil: ¿qué deberíamos hacer? Intervención Psicosocial, 3(9), 53-66. 
Vayreda, A., Tirado, F., \& Domènech, M. (2005). Construccionismo social, narratividad y simetría. En G. Limón (Comp.), Terapias Postmodernas. Aportaciones construccionistas (141-165). México: Editorial Pax.

White, M. (2004). El trabajo con personas que sufren las consecuencias del trauma múltiple. Desde la perspectiva narrativa. Recuperado de http://www.terapianarrativacoyoacan.com/ documentos/category/2-traducciones.html
Woodiwiss, J. (2014). Beyond a single story: The importance of separating 'harm' from 'wrongfulness' and 'sexual innocence' from 'childhood' in contemporary narratives of childhood sexual abuse. Sexualities, 17(1/2), 139-158.

World Health Organization. (2014, 05 de noviembre). Child Maltreatment. Recuperado de http://www.who.int/mediacentre/ factsheets/fs150/en/ 